\title{
LXIII. On quaternions; or on a new system of imaginaries in algebra
}

\author{
Sir William Rowan Hamilton LL.D. M.R.I.A. F.R.A.S.
}

To cite this article: Sir William Rowan Hamilton LL.D. M.R.I.A. F.R.A.S. (1849) LXIII. On quaternions; or on a new system of imaginaries in algebra, Philosophical Magazine Series 3, 34:231, 425-439, DOI: 10.1080/14786444908646261

To link to this article: http://dx.doi.org/10.1080/14786444908646261

册 Published online: 30 Apr 2009.

Submit your article to this journal $\lceil\pi$

Џll Article views: 5

Q View related articles $₫$

4 Citing articles: 1 View citing articles 주다. 
audition of the sounds emitted by separate points. Being referred to an admitted phænomenon, it is explained; and I think I may say that it had not been completely explained before.

The conclusion of these researches is, therefore, that the phænomena of simultaneous perception of several sounds pro. ceeding from the movement, whether of several points or of a single one, are only modifications of one general phænomenon, which may be stated in the following manner:-

"When our organ of hearing is affected by a movement that may be geometrically decomposed into several others, which, if they existed separately, would yield different sounds, we generally perceive all these sounds at the same time."

LXIII. On Quaternions; or on a Nerw System of Imaginaries in Algebra. By Sir William Rowan Hamilton, $L L . D$., M.R.I.A., F.R.A.S., Corresponding Member of the Institute of France, \&c., Andrews' Professor of Astronomy in the University of Dublin, and Royal Astronomer of Ireland.

[Continued from p. 343.]

71. REFORE entering on any discussion of this new form of the equation of the ellipsoid, namely the form

$$
\mathrm{TV} \frac{\eta \rho-\rho \theta}{\mathrm{U}(\eta-\theta)}=\theta^{2}-\eta^{2} \text {, eq. (159.), art. 70, }
$$

it may be useful to point out another manner of arriving at the same equation of the ellipsoid, by a different process of calculation, from that construction or generation of the surface, as the locus of the circle which is the mutual intersection of a pair of equal spheres, sliding within two fixed cylinders of revolution whose axes intersect each other; while the right line, connecting the centres of the two sliding spheres, moves parallel to itself, or remains constantly parallel to a fixed right line in the plane of the fixed axes of the cylinders: which mode of generating the ellipsoid was published in the Philosophical Magazine for July 1848 (having also been communicated to the Royal Irish Academy in the preceding May), as a deduction from the Calculus of Quaternions. And whereas the fixed right line, through the centre of the ellipsoid, to which the line connecting the centres of the two sliding spheres is parallel, may have either of two positions, since it may coincide with either of the two cyclic normals, we shall here suppose it to have the direction of the cyclic normal , or shall consider the second pair of sliding spheres 
mentioned in article 64 , of which the quaternion equations are, by article 62 (Phil. Mag. for July 1848),

$$
T(\rho-\mu)=T\left(\rho-\lambda^{\prime}\right)=b \text {. }
$$

72. Here (see Phíl. Mag. for May 1848), we have for $\mu$ the value,

and

$$
\mu=h^{\prime}(x-1) \text {, eq. (91.), art. 57; }
$$

also

$$
\lambda^{\prime}\left(x^{\prime}-l^{\prime}\right)=x^{\prime} \rho+\rho x^{\prime} \text {, eq. (110.), art. } 60 ;
$$

$$
\iota x^{\prime}=\iota^{\prime} x=\text { T. } \iota x \text {, eq. }(107) \text {, same article; }
$$

whence we derive for $\lambda^{\prime}$ the expression,

But

$$
\lambda^{\prime}=\frac{t^{-1} \rho+\rho t^{-1}}{t^{-1}-x^{-1}}=\frac{t \rho+\rho t}{1-t^{2} x^{-1}} . . . \text {. }
$$

$$
\left(1-\imath^{2} x^{-1}\right)^{-1}=\left\{l(x-1) x^{-1}\right\}^{-1}=x(x-1)^{-1} l^{-1} ; .
$$

and by (104.),

therefore

$$
p+p_{1}=-h^{\prime}(x-1)^{2} ; \quad . \quad . \quad .
$$

$$
\lambda^{\prime}=-h^{\prime} x(x-1) r^{-1}=h^{\prime}\left(x-x^{2} t^{-1}\right) . \quad \text {. . }
$$

If then we make, for abridgement,

$$
g=-h^{\prime} \mathrm{T} \frac{1-x}{\iota}, \cdot . \cdot \cdot
$$

and employ the two new fixed vectors $\eta$ and $\theta$, defined by the equations (see Phil. Mag. for May 1849),

$$
\eta=T_{\imath} U(b-x), \theta=T_{c} U\left(x^{-1}-s^{-1}\right),
$$

which have been found to give

$$
1-x=\eta \mathrm{T} \frac{1-x}{1}, \quad x-x^{2} 1^{-1}=-\theta \mathrm{T} \frac{1-x}{1},
$$

we shall have the values,

$$
\mu=g \eta ; \quad \lambda^{\prime}=g^{\theta} ; . . . . .
$$

and the lately cited equations (114.) of the two sliding spheres will become,

$$
\mathrm{T}(p-g \eta)=b ; \mathrm{T}(\rho-g \theta)=b ; . . .
$$

between which it remains to eliminate the scalar coefficient $g$, in order to find the equation of the ellipsoid, regarded as the locus of the circle in which the two spheres intersect each other.

73. Squaring the equations (146.), we find (by the general 
rules of this Calculus) for the two sliding spheres the two following more developed equations :

$$
\left.\begin{array}{l}
0=b^{2}+p^{2}-2 g \text { S. } n p+g^{2} \eta^{2} ; \\
0=b^{2}+p^{2}-2 g \text { S. } \theta p+g^{2} \theta^{2} .
\end{array}\right\} . . .
$$

Taking then the difference, and dividing by $g$, we find the equation

$$
g\left(\theta^{2}-\eta^{2}\right)=2 \mathrm{~S} .(\theta-\eta) \rho ; \quad . \quad . \quad .
$$

which, relatively to $\rho$, is linear, and may be considered as the equation of the plane of the varying circle of intersection of the two sliding spheres; any one position of that plane being distinguished from any other by the value of the coefficient $g$. Eliminating therefore that coefficient $g$, by substituting in (146.) its value as given by (148.), we find that the equation of the ellipsoid, regarded as the locus of the varying circle, may be presented under either of the two following new forms:

$$
\begin{aligned}
& \mathrm{T}\left(\rho-\frac{2 \eta \mathrm{S} .(\theta-\eta) p}{\theta^{2}-\eta^{2}}\right)=b ; . . . \\
& \mathrm{T}\left(\rho-\frac{2 \theta \mathrm{S} .(\eta-\theta) p}{\eta^{2}-\theta^{2}}\right)=b ; . . .
\end{aligned}
$$

respecting which two forms it deserves to be noticed, that either may be obtained from the other, by interchanging $\eta$ and $\theta$. And we may verify that these two last equations of the ellipsoid are consistent with each other, by observing that the semisum of the two vectors under the sign $T$ is perpendicular to their semidifference (as it ought to be, in order to allow of those two vectors themselves having any common length, such as $b)$; or that the condition of rectangularity,

$$
p-\frac{(\theta+\eta) S \cdot(\theta-\eta) p}{\theta^{2}-\eta^{2}} \perp \theta-\eta, \quad . \quad .
$$

is satisfied : which may be proved by showing (see Phil. Mag. for July 1846) that the scalar of the product of these two last vectors vanishes, as in fact it does, since the identity

$$
(\theta-\eta)(\theta+\eta)=\theta^{2}+\theta \eta-\eta \theta-\eta^{2},
$$

resolves itself into the two following formulæ:

$$
\left.\begin{array}{l}
\text { S. }(\theta-\eta)(\theta+\eta)=\theta^{2}-\eta^{2} ; \\
\text { V. }(\theta-\eta)(\theta+\eta)=\theta \eta-\eta^{\theta} ;
\end{array}\right\} \quad \text {. . . }
$$

of which the first is sufficient for our purpose. We may also verify the recent equations (149.) (150.) of the ellipsoid, by observing that they concur in giving the mean semiaxis $b$ as the length $T_{\rho}$ of the radius of that diametral and circular sec- 
tion, which is made by the cyclic plane having for equation

$$
\text { S. }(\theta-\eta)_{p}=0 \text {; . . . . . (153.) }
$$

this plane being found by the consideration that $\eta-\theta$ has the direction of the cyclic normal , or by making the coefficient $g=0$, in the formula (148.).

74. The equation (149.) of the ellipsoid may be successively transformed as follows :

$$
\begin{aligned}
b\left(\theta^{2}-\eta^{2}\right) & =\mathrm{T}\left\{\left(\theta^{2}-\eta^{2}\right) \rho-2 \eta \mathrm{S} .(\theta-\eta) \rho\right\} \\
& =\mathrm{T}\left\{\left(\theta^{2}-\eta^{2}\right) \rho-\eta(\theta-\eta) \rho-\eta \rho(\theta-\eta)\right\} \\
& =\mathrm{T}\left\{\theta^{2} \rho-\eta(\theta \rho+\rho \theta)+\eta \rho \eta\right\} \\
& =\mathrm{TV}\{(\theta-\eta) \theta \rho-\eta \rho(\theta-\eta)\} \\
& =\mathrm{TV} \cdot(\rho \theta-\eta \rho)(\theta-\eta) \\
& =\mathrm{TV} \cdot(\eta \rho-\rho \theta)(\eta-\theta) ; . . . .
\end{aligned}
$$

and by a similar series of transformations, performed on the equation (150.), we find also (remembering that $\theta^{2}-\eta^{2}$, being equal to $x^{2}-1^{2}$, is positive),

$$
b\left(\theta^{2}-\eta^{2}\right)=\mathrm{TV} \cdot(\rho \eta-\theta \rho)(\eta-\theta) .
$$

The same result (155.) may also be obtained by interchanging $\eta$ and $\theta$ in either of the two last transformed expressions (154.), for the positive product $b\left(\theta^{2}-\eta^{2}\right)$; and we may otherwise establish the agreement of these recent results, by observing that, in general, if $\mathbf{Q}$ and $\mathbf{Q}^{\prime}$ be any two conjugate quaternions (see Phil. Mag. for July 1846), such as are here $\eta \rho-\rho \theta$ and $\rho \eta-\theta p$, and if $\alpha$ be any vector, then

for

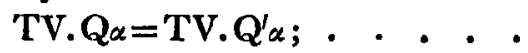

and because

$$
\left.\begin{array}{l}
V . Q_{\alpha}=\alpha S Q-V . \alpha V Q, \\
V \cdot Q^{\prime} \alpha=\alpha S Q+V . \alpha V Q ;
\end{array}\right\} \quad \cdot \quad \cdot
$$

$$
0=\mathrm{S} . \alpha \mathrm{V} . \alpha \mathrm{VQ}, \cdot \cdot \cdot \cdot(158 .)
$$

the common value of the two members of the formula (156.) is

$$
\text { TV.Q } \alpha=\sqrt{ }\left\{(\mathrm{TV} . \alpha \mathrm{VQ})^{2}+(\mathrm{T} \alpha . \mathrm{SQ})^{2}\right\} . \quad \text {. }
$$

If then we substitute for $b$ its value,

$$
b=\mathbf{T}(\eta-\theta) \text {, eq. (135.), art. } 70,
$$

and divide on both sides by this value of $\zeta$, we see, from (154.), (155.), that the equation of the ellipsoid may be put under either of these two other forms:

$$
\begin{aligned}
& \mathrm{TV} \cdot(\eta p-\rho \theta) \mathbf{U}(\eta-\theta)=\theta^{2}-\eta^{2}, \quad . \cdot \\
& \mathrm{TV} \cdot(\rho \eta-\theta \rho) \mathbf{U}(\eta-\theta)=\theta^{2}-\eta^{2} . \quad . \cdot
\end{aligned}
$$


But the versor of every vector is, in this calculus, a square root of negative unity; we have therefore in particular,

$$
(\mathrm{U}(\eta-\theta))^{2}=-1 \text {; . }
$$

and under the sign $T V$, as under the sign $T$, it is allowed to divide by -1 , without affecting the value of the tensor: it is therefore permitted to write the equation (160.) under the form

$$
\text { TV. } \frac{\eta p-p^{\theta}}{\mathrm{U}(\eta-\theta)}=\theta^{2}-\eta^{2},
$$

which form is thus demonstrated anew.

75. A few connected transformations may conveniently be noticed here. Since, for any quaternion $Q$,

$$
(\mathrm{TVQ})^{2}=-(\mathrm{VQ})^{2}=(\mathrm{TQ})^{2}-(\mathrm{SQ})^{2}
$$

while the tensor of a product is the product of the tensors, and the tensor of a versor is unity; and since

$$
\text { S. }(\rho \eta-\theta \rho)(\eta-\theta)=S\left(\rho \eta^{2}-\rho \eta \theta-\theta \rho \eta+\theta \rho \theta\right)=-2 S . \eta \theta \rho \text {, }
$$
because

$$
0=\mathbf{S} \cdot \rho \eta^{2}=\mathbf{S} \cdot \theta_{\rho} \theta \text {, and } \mathbf{S} \cdot \rho \eta \theta=\mathbf{S} \cdot \theta_{\rho} \eta=\mathbf{S} \cdot \eta \theta_{\rho} ; \quad \text {. }
$$

we have therefore, generally,

$$
\left.\begin{array}{l}
\mathbf{T} .(\rho \eta-\theta \rho) \mathbf{U}(\eta-\theta)=\mathbf{T}(\rho \eta-\theta \rho) ; \\
\text { S. }(\rho \eta-\theta \rho) \mathbf{U}(\eta-\theta)=-2 \mathrm{~T}(\eta-\theta)^{-1} \mathbf{S} . \eta \theta \rho ;
\end{array}\right\} .
$$

and there results the equation,

TV. $(\rho \eta-\theta \rho) \mathbf{U}(\eta-\theta)=\sqrt{ }\left\{\mathbf{T}(\rho \eta-\theta \rho)^{2}-4 \mathbf{T}(\eta-\theta)^{-2}(\mathbf{S} . \eta \theta \rho)^{2}\right\},(167$. as a general formula of transformation, valid for any three vectors, $\eta, \theta, \rho$. We may also, by the general rules of the present calculus, write the last result as follows,

$$
\begin{gathered}
\text { TV. }(p \eta-\theta p) \mathbf{U}(\eta-\theta)=\sqrt{ }\{(p \eta-\theta p)(\eta p-p \theta) \\
\left.\quad+(\eta-\theta)^{-2}(\eta \theta p-\rho \theta \eta)^{2}\right\} ; \quad . . . .
\end{gathered}
$$

the signs $S$ and $T$ thus disappearing from the expression of the radical. For the ellipsoid, this radical, being thus equal to the left-hand member of the formula (167.), or to that of (168.), must, by (161.), receive the constant value $\theta^{2}-\eta^{2}$; so that, by squaring on both sides, we find as a new form of the equation (161.) of the ellipsoid, the following:

$$
\left(\theta^{2}-\eta^{2}\right)^{2}=\left(\rho \eta-\theta_{\rho}\right)\left(\eta p-\rho^{\theta}\right)+(\eta-\theta)^{-2}\left(\eta \theta_{\rho}-\rho \theta \eta\right)^{2} \ldots
$$

Or, by a partial reintroduction of the signs $S$ and $T$, we find this somewhat shorter form:

$$
\Gamma(\rho \eta-\theta \rho)^{2}+4(\eta-\theta)^{-2}(\mathrm{~S} . \eta \hat{\theta} \rho)^{2}=\left(\theta^{2}-\eta^{2}\right)^{2} .
$$


And instead of the square of the tensor of the quaternion $\rho \eta-\theta \rho$, we may write any one of several general expressions for that square, which will easily suggest themselves to those who have studied the transformations (already printed in this Magazine), of the earlier and in some respects simpler equation of the ellipsoid, proposed by the present writer, namely the equation

$$
\mathbf{T}(\iota p+\rho x)=x^{2}-\iota^{2} \text {. eq. (9.), art. } 38 .
$$

For instance, we may employ any of the following general equalities, which all flow with little difficulty from the principles of the present calculus :

$$
\begin{aligned}
& \mathbf{T}(\rho \eta-\theta p)^{2}=\mathbf{T}\left(\eta p-\rho^{\theta}\right)^{2} \\
& =(p \eta-\theta p)\left(\eta p-p^{\theta}\right)=\left(\eta p-p^{\theta}\right)(p \eta-\theta p) \\
& =\left(\eta^{2}+\theta^{2}\right) \rho^{2}-\rho \eta p \theta-\theta \rho \eta \rho \\
& =\left(\eta^{2}+\theta^{2}\right) \rho^{2}-\eta \rho \theta p-p \theta p \eta \\
& =(\eta+\theta)^{2} \rho^{2}-(\eta p+\rho \eta)(\theta p+\rho \theta) \\
& =\left(\eta^{2}+\theta^{2}\right) \rho^{2}-2 \mathrm{~S} \text {. } \eta \rho \theta \rho \\
& =(\eta+\theta)^{2} \rho^{2}-4 \text { S. } \eta p \cdot S \cdot \theta \rho \\
& =(\eta-\theta)^{2} \rho^{2}+4 \cdot S(V \cdot \eta p \cdot V \cdot p \theta) ; \quad .
\end{aligned}
$$

and which all hold good, independently of any relation between the three vectors $\eta, \theta, \rho$.

76. As bearing on the last of these transformations it seems not useless to remark, that a general formula published in the Philosophical Magazine of August 1846, for any three vectors $\alpha, \alpha^{\prime}, \alpha^{\prime \prime}$, namely the formula

$\alpha \mathrm{S} . \alpha^{\prime} \alpha^{\prime \prime}-\alpha^{\prime} \mathrm{S} . \alpha^{\prime \prime} \alpha=\mathrm{V}\left(\mathrm{V}, \alpha \alpha^{\prime} \cdot \alpha^{\prime \prime}\right)$, eq. (12.) of art 22 ,

which is found to be extensively useful, and indeed of constant recurrence in the applications of the calculus of quaternions, may be proved symbolically in the following way, which is shorter than that employed in the 23rd article:

$$
\begin{aligned}
& \mathrm{V}\left(\mathrm{V} \cdot \alpha \alpha^{\prime} \cdot \alpha^{\prime \prime}\right)=\frac{1}{2}\left(\mathrm{~V} \cdot \alpha \alpha^{\prime} \cdot \alpha^{\prime \prime}-\alpha^{\prime \prime} \mathrm{V} \cdot \alpha \alpha^{\prime}\right)=\frac{1}{2}\left(\alpha \alpha^{\prime} \cdot \alpha^{\prime \prime}-\alpha^{\prime \prime} \cdot \alpha \alpha^{\prime}\right) \\
& \quad=\frac{1}{2} \alpha\left(\alpha^{\prime} \alpha^{\prime \prime}+\alpha^{\prime \prime} \alpha^{\prime}\right)-\frac{1}{2}\left(\alpha \alpha^{\prime \prime}+\alpha^{\prime \prime} \alpha\right) \alpha^{\prime}=\alpha \mathrm{S} \cdot \alpha^{\prime} \alpha^{\prime \prime}-\alpha^{\prime} \mathbf{S} \cdot \alpha^{\prime \prime} \alpha .
\end{aligned}
$$

The formula may be also written thus:

$$
\text { V. } \alpha^{\prime \prime} \text { V. } \alpha^{\prime} \alpha=\alpha \text { S. } \alpha^{\prime} \alpha^{\prime \prime}-\alpha^{\prime} \mathrm{S} . \alpha \alpha^{\prime \prime} ; . . .
$$

whence easily flows this other general and useful transformation, for the vector part of the product of any three vectors, $\alpha, \alpha^{\prime}, \alpha^{\prime \prime}$ :

$$
\text { V. } \alpha^{\prime \prime} \alpha^{\prime} a=\alpha \text { S. } \alpha^{\prime} \alpha^{\prime \prime}-\alpha^{\prime} \mathrm{S} \cdot \alpha^{\prime \prime} \alpha+\alpha^{\prime \prime} \mathrm{S} . \alpha \alpha^{\prime} \text {. }
$$

Operating on this by S. $\alpha^{\prime \prime \prime}$, we find, for the scalar part of the product of any four vectors, the expression :

S. $\alpha^{\prime \prime \prime} \alpha^{\prime \prime} \alpha^{\prime} \alpha=$ S. $\alpha^{\prime \prime \prime} \alpha . S . \alpha^{\prime} \alpha^{\prime \prime}-S \cdot \alpha^{\prime \prime \prime} \alpha^{\prime} . S . \alpha^{\prime \prime} \alpha+$ S. $\alpha^{\prime \prime \prime} \alpha^{\prime \prime} . S . \alpha \alpha^{\prime} .(175$. 
But a quaternion, such as is $\alpha^{\prime} \alpha$ or $\alpha^{\prime \prime \prime} \alpha^{\prime \prime}$, is always equal to the sum of its own scalar and vector parts; and the product of a scalar and a vector is a vector, while the scalar of a vector is zero : therefore and

$$
\alpha^{\prime} \alpha=S . \alpha^{\prime} \alpha+V . \alpha^{\prime} \alpha, \quad \alpha^{\prime \prime \prime} \alpha^{\prime \prime}=\mathrm{S} . \alpha^{\prime \prime \prime} \alpha^{\prime \prime}+\mathrm{V} . \alpha^{\prime \prime \prime} \alpha^{\prime \prime},
$$

$$
\text { S. } \alpha^{\prime \prime \prime} \alpha^{\prime \prime} \alpha^{\prime} \alpha=\mathrm{S} . \alpha^{\prime \prime \prime} \alpha^{\prime \prime} . \mathrm{S} . \alpha^{\prime} \alpha+\mathrm{S}\left(\mathrm{V} \cdot \alpha^{\prime \prime \prime} \alpha^{\prime \prime} . \mathrm{V} \cdot \alpha^{\prime} \alpha\right) \text {. }
$$

Comparing then (175.) and (177.), and observing that

$$
\text { S. } \alpha \alpha^{\prime}=+\mathrm{S} . \alpha^{\prime} \alpha, \quad \mathrm{V}, \alpha \alpha^{\prime}=-\mathrm{V} . \alpha^{\prime} \alpha, \quad \text {. }
$$

we obtain the following general expression for the scalar part of the product of the vectors of any two binary products of vectors :

$$
\mathrm{S}\left(\mathrm{V} \cdot \alpha^{\prime \prime \prime} \alpha^{\prime \prime} . \mathrm{V} . \alpha^{\prime} \alpha\right)=\mathrm{S} . \alpha^{\prime \prime \prime} \alpha . \mathrm{S}, \alpha^{\prime} \alpha^{\prime \prime}-\mathrm{S} . \alpha^{\prime \prime \prime} \alpha^{\prime} . \mathrm{S} . \alpha^{\prime \prime} \alpha ;
$$

while the vector part of the same product of vectors is easily found, by similar processes, to admit of being expressed in either of the two following ways (compare equation (3.) of article 24):

$$
\begin{aligned}
\mathrm{V}\left(\mathrm{V} . \alpha^{\prime \prime \prime} \alpha^{\prime \prime} . \mathrm{V} . \alpha^{\prime} \alpha\right) & =\alpha^{\prime \prime \prime} \mathrm{S} . \alpha^{\prime \prime} \alpha^{\prime} \alpha-\alpha^{\prime \prime} \mathrm{S} . \alpha^{\prime \prime \prime} \alpha^{\prime} \alpha \\
& =\alpha \mathrm{S} . \alpha^{\prime \prime \prime} \alpha^{\prime \prime} \alpha^{\prime}-\alpha^{\prime} \mathrm{S} . \alpha^{\prime \prime \prime} \alpha^{\prime \prime} \alpha ; . . .
\end{aligned}
$$

of which the combination conducts to the following general expression for any fourth vector $\boldsymbol{\alpha}^{\prime \prime \prime}$, or $\rho$, in terms of any three given vectors $\alpha, \alpha^{\prime}, \alpha^{\prime \prime}$, which are not parallel to any one common plane (compare equation (4.) of article 26):

$$
\rho S . \alpha^{\prime \prime} \alpha^{\prime} \alpha=\alpha S \cdot \alpha^{\prime \prime} \alpha^{\prime} p+\alpha^{\prime} \mathrm{S}, \alpha^{\prime \prime} p \alpha+\alpha^{\prime \prime} \mathrm{S} . \alpha^{\prime} \alpha, \quad .
$$

If we further suppose that

we shall have

$$
\alpha^{\prime \prime}=\mathrm{V} \cdot \alpha^{\prime} \alpha, \quad . \quad . . .
$$

$$
\text { S. } \alpha^{\prime \prime} \alpha^{\prime} \alpha=\left(\mathrm{V} . \alpha^{\prime} \alpha\right)^{2}=\alpha^{\prime \prime 2} \text {; . . . }
$$

and after dividing by $\alpha^{\prime \prime 2}$, the recent equation (181.) will become

$$
\rho=\alpha \mathrm{S} \frac{\alpha^{\prime} \rho}{\alpha^{\prime \prime}}+\alpha^{\prime} \mathrm{S} \frac{\rho \alpha}{\alpha^{\prime \prime}}+\frac{\mathrm{S} \cdot \alpha^{\prime \prime} \rho}{\alpha^{\prime \prime}} 3 . .
$$

whereby an arbitrary vector $p$ may be expressed, in terms of any two given vectors $\alpha, \alpha^{\prime}$ which are not parallel to any common line, and of a third vector $\alpha^{\prime \prime}$, perpendicular to both of them. And if, on the other hand, we change $\alpha, \alpha^{\prime}, \alpha^{\prime \prime}, \alpha^{\prime \prime \prime}$ to $\theta, \rho, \rho, \eta$, in the general formula (179.), we find that generally, for any three vectors $\eta, \theta, \rho$, the following identity holds good:

$$
S\left(V . \eta \rho . V . \rho^{\theta}\right)=\rho^{2} \mathbf{S} \cdot \eta \theta-S . \eta p \cdot S \cdot \rho^{\theta} ; \quad \text {. }
$$

which serves to connect the two last of the expressions (171.), and enables us to transform either into the other. 
77. To show the geometrical meaning of the equation (185.), let us divide it on both sides by T. $\rho^{2} y \theta$; it then becomes, after transposing,

$$
-\mathrm{SU} \cdot \eta^{\theta}=\mathrm{SU} \cdot \eta \rho \cdot \mathrm{SU} \cdot \rho^{\theta}+\mathrm{S}\left(\mathrm{VU} \cdot \eta \rho \cdot \mathrm{VU} \cdot \rho^{\theta}\right) .
$$

Here, by the general principles of the geometrical interpretation of the symbols employed in this calculus (see the remarks in the Philosophical Magazine for July 1846), the symbol SU. $\eta^{\theta}$ is an expression for the cosine of the supplement of the angle between the two arbitrary vectors $\eta$ and $\theta$; and therefore the symbol $-S U \cdot \eta \theta$ is an expression for the cosine of that angle itself. In like manner, $-\mathrm{SU}$. $\eta p$ and $-\mathrm{SU} . \rho \theta$ are expressions for the cosines of the respective inclinations of those two vectors $\eta$ and $\theta$ to a third arbitrary vector $\rho$; and at the same time VU. $\eta p$ and $V U . \rho \theta$ are vectors, of which the lengths represent the sines of the same two inclinations last mentioned, while they are directed towards the poles of the two positive rotations corresponding; namely the rotations from $\eta$ to $\rho$, and from $\rho$ to $\theta$, respectively. The vectors VU. $p$ and VU. $\rho^{\theta}$ are therefore inclined to each other at an angle which is the supplement of the dihedral or spherical angle, subtended at the unit-vector $\mathrm{U}_{\rho}$, or at its extremity on the unit-sphere, by the two other unit-vectors $U_{\eta}$ and $U \theta$, or by the arc between their extremities: so that the scalar part of their product, in the formula (186.), represents the cosine of this spherical angle itself (and not of its supplement), multiplied into the product of the sines of the two sides or arcs upon the sphere, between which that angle is included. If then we denote the three sides of the spherical triangle, formed by the extremities of the three unit-vectors $\mathrm{U}_{\eta}, \mathrm{U} \theta, \mathrm{U}_{\rho}$, by the symbols, $\overparen{\eta}, \widehat{\eta} \rho, \widehat{\rho}_{\hat{\theta}}$, and the spherical angle opposite to the first of them by the symbol $x p \theta$, the equation (186.) will take the form

$$
\cos \widehat{\eta \theta}=\cos \widehat{\eta \rho} \cos \widehat{\rho \theta}+\sin \widehat{\eta \rho} \sin \widehat{\rho} \cos \hat{\eta \rho \theta} \text {; }
$$

which obviously coincides with the well-known and fundamental formula of spherical trigonometry, and is brought forward here merely as a verification of the consistency of the results of this calculus, and as an example of their geometrical interpretability.

A more interesting example of the same kind is furnished by the general formula (179.) for four vectors, which, when divided by the tensor of their product, becomes

$$
\begin{gathered}
\mathrm{S}\left(\mathrm{VU}, \alpha^{\prime \prime \prime} \alpha^{\prime \prime} . \mathrm{VU}, \alpha^{\prime} \alpha\right)=\mathrm{SU} . \alpha^{\prime \prime \prime} \alpha . \mathrm{SU} \cdot \alpha^{\prime} \alpha^{\prime \prime} \\
-\mathrm{SU}, \alpha^{\prime \prime \prime} \alpha^{\prime} . \mathrm{SU} \cdot \alpha^{\prime \prime} \alpha ; . . . .
\end{gathered}
$$


and signifies, when interpreted on the same principles, that

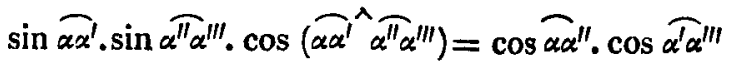

$$
\begin{aligned}
& -\cos \widetilde{\alpha \alpha^{\prime \prime \prime}} \cdot \cos \widetilde{\alpha^{\prime} \alpha^{\prime \prime}} \text {; }
\end{aligned}
$$

where the spherical angle between the two arcs from $\alpha$ to $\alpha^{\prime}$ and from $\alpha^{\prime \prime}$ to $\alpha^{\prime \prime \prime}$ may be replaced by the interval between the poles of the two positive rotations corresponding. The same result may be otherwise stated as follows: If $L, L, L^{\prime \prime}, L^{\prime \prime \prime}$, denote any four points upon the surface of an unit-sphere, and $A$ the angle which the arcs $L L, L^{\prime \prime} L^{\prime \prime}$ form where they meet each other, (the arcs which include this angle being measured in the directions of the progressions from $L$ to $L^{\prime}$, and from $L^{\prime \prime}$ to $L^{\prime \prime \prime}$ respectively, then the following equation will hold good :

$$
\begin{gathered}
\cos L L^{\prime \prime} \cdot \cos L^{\prime} L^{\prime \prime \prime}-\cos L L^{\prime \prime \prime} \cdot \cos L^{\prime} L^{\prime \prime} \\
=\sin L L^{\prime} \cdot \sin L^{\prime \prime} L^{\prime \prime \prime} \cdot \cos A .
\end{gathered}
$$

Accordingly this last equation has been incidentally given, as an auxiliary theorem or lemma, at the commencement of those profound and beautiful researches, entitled Disquisitiones Generales circa Superficies Curvas, which were published by Gauss at Göttingen in 1828. That great mathematician and philosopher was content to prove the last written equation by the usual formulæ of spherical and plane trigonometry; but, however simple and elegant may be the demonstration thereby afforded, it appears to the present writer that something is gained by our being able to present the result (190.) or (189.), under the form (188.) or (179.), as an identity in the quaternion calculus. In general, all the results of plane and spherical trigonometry take the form of identities in this calculus; and their expressions, when so obtained, are associated with a reference to vectors, which is usually suggestive of graphic as well as metric relations.

78. Since

$$
\rho \eta-\theta p=S \cdot \rho(\eta-\theta)+\mathrm{V} \cdot \rho(\eta+\theta), \quad \cdot \cdot
$$

the quaternion $\rho \eta-\theta \rho$ gives a pure vector as a product, or as a quotient, if it be multiplied or divided by the vector $\eta+\theta$ (compare article 68); we may therefore write

$$
p \eta-\theta p=\lambda_{1}(\eta+\theta), \cdot . \cdot \cdot
$$

$\lambda_{1}$ being a new vector-symbol, of which the value may be thus expressed :

$$
\lambda_{1}=p-2(\eta+\theta)^{-1} \mathrm{~S} .9 p \text {. . . . . }
$$

Phil. Mag. S, 3. Vol. 34. No. 231. June 1849. 2 F 
The equation (192.) will then give,

$$
\left.\begin{array}{l}
\mathbf{T}(p \eta-\theta p)=\mathbf{T} \lambda_{1} \cdot T(\eta+\theta) ; \\
\mathbf{T}(p \eta-\theta p)^{2}=\lambda_{1}^{2}(\eta+\theta)^{2} .
\end{array}\right\} . .
$$

We have also the identity,

$$
\left(\theta^{2}-\eta^{2}\right)^{2}=(\eta-\theta)^{2}(\eta+\theta)^{2}+(\eta \theta-\theta \eta)^{2} ; \quad \cdot
$$

which may be shown to be such, by observing that

$$
\begin{aligned}
(\eta-\theta)^{2}(\eta+\theta)^{2}=\left(\eta^{2}+\theta^{2}-2 \mathrm{~S} \cdot \eta \theta\right)\left(\eta^{2}+\theta^{2}+2 \mathrm{~S} \cdot \eta \theta\right) & \\
& =\left(\eta^{2}+\theta^{2}\right)^{2}-4(\mathrm{~S} \cdot \eta \theta)^{2}=\left(\eta^{2}-\theta^{2}\right)^{2}+4(\mathrm{~T} \cdot \eta \theta)^{2}-4 \cdot(\mathrm{S} \cdot \eta \theta)^{2} \\
& =\left(\eta^{2}-\theta^{2}\right)^{2}-4(\mathrm{~V} \cdot \eta \theta)^{2}=\left(\theta^{2}-\eta^{2}\right)^{2}-(\eta \theta-\theta \eta)^{2} ; . \quad . \quad(1
\end{aligned}
$$

or by remarking that (see equations (152.)(163.)),

$$
\left.\begin{array}{c}
\eta^{2}-\theta^{2}=\mathrm{S} \cdot(\eta-\theta)(\eta+\theta), \quad \eta \theta-\theta \eta=\mathrm{V} \cdot(\eta-\theta)(\eta+\theta), \\
\quad \text { and }(\eta-\theta)^{2}(\eta+\theta)^{2}=(\mathrm{T} \cdot(\eta-\theta)(\eta+\theta))^{2} ;
\end{array}\right\}
$$

or in several other ways. Introducing then a new vector $\varepsilon$, such that

$$
\eta \theta-\theta \eta=\varepsilon \mathbf{T}(\eta+\theta), \text { or, } \varepsilon=2 \mathrm{~V} \cdot \eta \theta . \mathbf{T}(\eta+\theta)^{-1} ; .
$$

and that therefore

and

$$
(\eta \theta-\theta \eta)^{2}=-\varepsilon^{2}(\eta+\theta)^{2}, \ldots . . .
$$

$2 \mathrm{~S} \cdot \eta \theta_{\rho}=\mathrm{S} \cdot \varepsilon_{\rho} \cdot \mathrm{T}(\eta+\theta), 4\left(\mathrm{~S} \cdot \eta \theta_{\rho}\right)^{2}=-\left(\mathrm{S} . \varepsilon_{\rho}\right)^{2}(\eta+\theta)^{2} ;(200$. while, by (135.),

$$
\mathbf{T}(\eta-\theta)=b, \quad(\eta-\theta)^{2}=-b^{2} ;
$$

we find that the equation (170) of the ellipsoid, after being divided by $(\eta+\theta)^{2}$, assumes the following form :

$$
\lambda_{1}^{2}+b^{-2}(\text { S. } s p)^{2}+b^{2}+\varepsilon^{2}=0 . \quad . \quad .
$$

But also, by (193.), (198.),

$$
\text { S. } \varepsilon \lambda_{1}=\mathrm{S} . \varepsilon \rho ; \quad \cdot \text {. }
$$

the equation (202.) may therefore be also written thus:

$$
0=\left(\lambda_{1}-\varepsilon\right)^{2}+\left(b+b^{-1} S \cdot \varepsilon \rho\right)^{2} ;
$$

and the scalar $b+b^{-1}$ S.ep is positive, even at an extremity of the mean axis of the ellipsoid, because, by (295.) (199.) (201.), we have

$$
\left(\theta^{2}-\eta^{2}\right)^{2}=-\left(b^{2}+\varepsilon^{2}\right)(\eta+\theta)^{2}=\left(b^{2}-\mathrm{T} \varepsilon^{2}\right) \mathrm{T}(\eta+\theta)^{2}, \quad .
$$
and therefore

$$
\mathrm{T} \varepsilon<6 \text {. . . . . . (206.) }
$$

We have then this new form of the equation of the ellipsoid, deduced by transposition and extraction of square roots 
(according to the rules of the present calculus), from the form (204.):

$$
T\left(\lambda_{1}-\varepsilon\right)=b+b^{-1} \text { S.sp. . . . . }
$$

By a process exactly similar to the foregoing, we find also the form

$$
T\left(\lambda_{1}+\varepsilon\right)=b-b^{-1} \text { S.ę; . . . }
$$

which differs from the equation last found, only by a change of sign of the auxiliary and constant vector $\varepsilon$ : and hence, by addition of the two last equations, we find still another form, namely,

$$
T\left(\lambda_{1}-\varepsilon\right)+T\left(\lambda_{1}+\varepsilon\right)=2 b ; \quad . \quad . \quad .
$$

or substituting for $\lambda_{1}$, $\varepsilon$, and $b$ their values, in terms of $\eta, \theta$, and $\rho$, and multiplying into $T(\eta+\theta)$,

$$
\begin{aligned}
& \mathrm{T}\left(\frac{p \eta-\theta p}{\mathrm{U}(\eta+\theta)}-2 \mathrm{~V} \cdot \eta \theta\right)+\mathrm{T}\left(\frac{p \eta-\theta p}{\mathrm{U}(\eta+\theta)}+2 \mathrm{~V} \cdot \eta \theta\right) \\
& \quad=2 \mathrm{~T} \cdot(\eta-\theta)(\eta+\theta) . . . . . . . .
\end{aligned}
$$

79. The locus of the termination of the auxiliary and variable vector $\lambda_{1}$, which is derived from the vector $\rho$ of the original ellipsoid by the linear formula (193.), is expressed or represented by the equation (209.); it is therefore evidently a certain nere ellipsoid, namely an ellipsoid of revolution, which has the mean axis $2 b$ of the old or given ellipsoid for its major axis, or for its axis of revolution, while the vectors of its two foc $i$ are denoted by the symbols $+\varepsilon$ and $-\varepsilon$. If $a$ denote the greatest, and $c$ the least semiaxis, of the original ellipsoid, while $b$ still denotes its mean semiaxis, then, by what has been shown in former articles, we have the values,

$$
\mathrm{T}_{\eta}=\mathrm{T}_{\iota}=\frac{1}{2}(a+c) ; \quad \mathrm{T}^{\theta}=\mathrm{T}_{x}=\frac{1}{2}(a-c) ; .
$$

and consequently (compare the note to art. 70),

therefore

$$
a=\mathrm{T}_{\eta}+\mathrm{T} \theta ; \quad c=\mathrm{T}_{\eta}-\mathrm{T} \theta ; . . .
$$

also

$$
a c=\Gamma \eta^{2}-\mathrm{T} \theta^{2}=\theta^{2}-\eta^{2} ; . . . .
$$

and

$$
\begin{gathered}
\mathrm{T}(\eta+\theta)^{2}+b^{2}=-(\eta+\theta)^{2}-(\eta-\theta)^{2}=-2 \eta^{2}-2 \theta^{2} \\
=2 \mathrm{~T} \eta^{2}+2 \mathrm{~T} \theta^{2}=(\mathrm{T} \eta+\mathrm{T} \theta)^{2}+\left(\mathrm{T}_{\eta}-\mathrm{T} \theta\right)^{2},
\end{gathered}
$$

whence, by (205.),

$$
\mathrm{T}(\eta+\theta)^{2}=a^{2}-b^{2}+c^{2} ; . . .
$$

$$
\mathrm{T}_{\epsilon}^{2}=b^{2}-\frac{a^{2} c^{2}}{a^{2}-b^{2}+c^{2}}=\frac{\left(a^{2}-b^{2}\right)\left(b^{2}-c^{2}\right)}{a^{2}-b^{2}+c^{2}} .
$$

2 F 2 
Such, then, is the expression for the square of the distance of either focus of the new or derived ellipsoid of revolution, which has $\lambda_{1}$ for its varying vector, from the common centre of the new and old ellipsoids, which centre is also the common origin of the vectors $\lambda_{1}$ and $\rho$ : while these two foci of the new ellipsoid are situated upon the mean axis of the old one. There exist also other remarkable relations, between the original ellipsoid with three unequal semiaxes $a, b, c$, and the new ellipsoid of revolution, of which some will he brought into view, by pursuing the quaternion analysis in a way which we shall proceed to point out.

80. The geometrical construction already mentioned (in articles $64,71,8 c$.), of the original ellipsoid as the locus of the circle in which two sliding spheres intersect, shows easily (see art. 72) that the scalar coefficient $g$, in the equations (146.) of that pair of sliding spheres, becomes equal to the number 2 , at one of those limiting positions of the pair, for which, after cutting, they touch, before they cease to meet each other. In fact, if we thus make

$$
g=2, \text {. . . . . . . }
$$

the values (145.) of the vectors of the centres will give, for the interval between those two centres of the two sliding spheres, the expression

$$
\mathrm{T}\left(\mu-\lambda^{\prime}\right)=g^{\prime} \mathrm{T}(\eta-\theta)=2 b ; . . . \quad .
$$

this interval will therefore be in this case equal to the diameter of either sliding sphere, because it will be equal to the mean axis of the ellipsoid: and the two spheres will touch one another. Had we assumed a value tor $g$, less by a very little than the number 2 , the two spheres would have cut each other in a very small circle, of which the circumference would have been (by the construction) entirely contained upon the surface of the ellipsoid; and the plane of this little circle would have been parallel and very near to that other plane, which was the cornmon tangent plane of the two spheres, and also of the ellipsoid, when $g$ received the value $z$ itself. It is clear, then, that this value 2 of $g$ corresponds to an umbilicar point on the ellipsoid; and that the equation

$$
\text { S. }(\theta-\eta) p=\theta^{2}-\eta^{2}
$$

which is obtained from the more general equation (148.) of the plane of a circle on the ellipsoid, by changing $g$ to 2 , represents an umbilicar tangent plane, at which the normal has the direction of the vector $\eta-\theta$. Accordingly it has been seen that this last vector has the direction of the cyclic normal ; in fact, the expressions (131.), for $\eta$ and $\theta$ in terms of, and 
$x$, give conversely these other expressions for the latter vectors in terms of the former,

$$
\imath=\mathrm{T}_{\eta} \mathrm{U}(\eta-\theta) ; \quad x=\mathrm{T} \theta \mathrm{U}\left(\theta^{-1}-\eta^{-1}\right): \text {. }
$$

whence (it may here be noted) follow the two parallelisms,

$$
\begin{aligned}
& \mathrm{U}_{1}-\mathrm{U}_{x}=\mathrm{U}(\eta-\theta)+\mathrm{U}\left(\eta^{-1}-\theta^{-1}\right) \| \mathrm{U}_{\eta}+\mathrm{U} \theta ; . \\
& \mathrm{U}_{1}+\mathrm{U}_{x}=\mathrm{U}(\eta-\theta)-\mathrm{U}\left(\eta^{-1}-\theta^{-1}\right) \| \mathrm{U}_{\eta}-\mathrm{U}^{\theta} ; .
\end{aligned}
$$

the members of (221.) having each the direction of the greatest axis of the ellipsoid, and the members of (222.) having each the direction of the least axis ; as may easily be proved, for the first members of these formulæ, by the construction with the diacentric sphere, which was communicated by the writer to the Royal Irish Academy in 1846, and was published in the present Magazine in the course of the following year. The equation (219.) may be verified by observing that it gives, for the length of the perpendicular let fall from the centre of the ellipsoid on an umbilicar tangent plane, the expression

$$
p=\left(\theta^{2}-\eta^{2}\right) \mathrm{T}(\eta-\theta)^{-1}=a c b^{-1} ; \quad . \quad .
$$

agreeing with known results. And the vector $\omega$ of the umbilicar point itself must be the semisum of the vectors of the centres of the two equal and sliding spheres, in that limiting position of the pair in which (as above) they touch each other; this umbilicar vector $\omega$ is therefore expressed as follows:

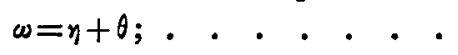

because this is the semisum of $\mu$ and $\lambda^{\prime}$ in (145.), or of $g \eta$ and $g \theta$ when $g=2$. (Compare the note to article 70.) As a verification, we may observe that this expression (224.) gives, by (215.), the following known value for the length of an umbilicar semidiameter of the ellipsoid,

$$
u=\mathrm{T} \omega=\mathrm{T}(\eta+\theta)=\sqrt{ }\left(a^{2}-b^{2}+c^{2}\right) . .
$$

By similar reasonings it may be shown that the expression

$$
\omega^{\prime}=\mathrm{T}_{\eta} \mathrm{U} \theta+\mathrm{T} \theta \mathrm{U} \eta, \quad \text {. . . . (226.) }
$$

which may also be thus written, (see same note to art. 70,)

$$
\omega^{\prime}=-\mathrm{T} \cdot \eta \theta \cdot\left(\eta^{-1}+\theta^{-1}\right)
$$

represents another umbilicar vector; in fact, we have, by (224.) and (226.),

and

$$
\top \omega^{\prime}=\mathbf{T} \omega, \quad \text {. . . . . . }
$$

$$
\left.\begin{array}{l}
\omega+\omega^{\prime}=\left(\mathrm{T}_{\eta}+\mathrm{T} \theta\right)\left(\mathrm{U}_{\eta}+\mathrm{U} \theta\right), \\
\omega-\omega^{\prime}=\left(\mathrm{T}_{\eta}-\mathrm{T} \theta\right)\left(\mathrm{U}_{\eta}-\mathrm{U} \theta\right) ;
\end{array}\right\} . . .
$$

so that the vectors $\omega \omega^{\prime}$ are equally long, and the angle between 
them is bisected by $\mathrm{Ur}+\mathrm{U} \theta$, or (see (221.)) by the axis major of the ellipsoid; while the supplementary angle between $\omega$ and $-\omega$ is bisected by $\mathrm{U}_{\eta}-\mathrm{U} \theta$, or (as is shown by (222.)) by the axis minor. It is evident that $-\omega$ and $-\omega^{\prime}$ are also umbilicar vectors; and it is clear, from what has been shown in former articles, that the vectors $\eta$ and $\theta$ have the directions of the axes of the two cylinders of revolution, which can be circumscribed about that given or original ellipsoid, to which all the remarks of the present article relate.

81. These remarks being premised, let us now resume the consideration of the variable vector $\lambda_{1}$, of art. 78, which has been seen to terminate on the surface of a certain derived ellipsoid of revolution. Writing, under a slightly altered form, the expression (193.) for that vector $\lambda_{1}$, and combining with it three other analogous expressions, for three other vectors, $\lambda_{2}, \lambda_{3}, \lambda_{4}$, as follows,

$$
\left.\begin{array}{ll}
\lambda_{1}=\frac{p \eta-\theta p}{\eta+\theta} ; & \lambda_{2}=\frac{p \theta-\eta p}{\eta+\theta} ; \\
\lambda_{3}=\frac{p^{\theta-1}-\eta^{-1} p}{\eta^{-1}+\theta^{-1}} ; & \lambda_{4}=\frac{p \eta^{-1}-\theta^{-1} p}{\eta^{-1}+\theta^{-1}} ;
\end{array}\right\}
$$

it is easy to prove that

and that

$$
\mathrm{T} \lambda_{1}=\mathrm{T} \lambda_{2}=\mathrm{T} \lambda_{3}=\mathrm{T} \lambda_{4} ; . . . .
$$

$$
\text { S. } \eta \theta \lambda_{1}=S . \eta \theta \lambda_{2}=S . \eta \theta \lambda_{3}=S . \eta \theta \lambda_{4}=S \cdot \eta \theta p ; \quad \text {. }
$$

whence it follows that the four vectors $\lambda_{1}, \lambda_{2}, \lambda_{3}, \lambda_{4}$, being supposed to be all drawn from the centre $\Lambda$ of the original ellipsoid, terminate in four points, $\mathbf{L}_{1}, \mathbf{L}_{2}, \mathbf{L}_{3}, \mathbf{L}_{4}$, which are the corners of a quadrilateral inscribed in a circle of the derived ellipsoid of revolution; the plane of this circle being parallel to the plane of the greatest and least axes of the original ellipsoid, and passing through the point $\mathrm{E}$ of that ellipsoid, which is the termination of the vector $\rho$. We shall have also the equations,

$$
\frac{\lambda_{2}-p}{\lambda_{1}-p}=\frac{\mathrm{S} \cdot \eta p}{\mathrm{~S} \cdot \theta_{p}}=\mathrm{V}^{-1} 0 ; \frac{\lambda_{3}-p}{\lambda_{4}-p}=\frac{\mathrm{S} \cdot \eta^{-\mathrm{I}} p}{\mathrm{~S} \cdot \theta^{-1} p}=\mathrm{V}^{-1} 0 ;
$$

which show that the two opposite sides $\mathrm{L}_{1} \mathrm{~L}_{2}, \mathrm{~L}_{3} \mathrm{~L}_{4}$, of thisinscribed quadrilateral, being prolonged if necessary, intersect in the lately-mentioned point $\mathbf{E}$ of the original ellipsoid. And because the expressions (230.) give also

$$
\mathrm{V} \frac{\lambda_{2}-\lambda_{1}}{\eta+\theta}=0, \quad \mathrm{~V} \frac{\lambda_{4}-\lambda_{3}}{\eta^{-1}+\theta^{-1}}=0, \quad .
$$

these opposite sides $\mathbf{L}_{1} \mathbf{L}_{2}, \mathbf{L}_{3} \mathbf{L}_{4}$, of the plane quadrilateral thus 
inscribed in a circle of the derived ellipsoid of revolution, are parallel respectively to the vectors $\eta+\theta, \eta^{-1}+\theta^{-1}$, or to the two umbilicar vectors $\omega, \omega^{\prime}$, of the original ellipsoid, with the semiaxes abc. At the same time, the equations

$$
\mathrm{V} \frac{\lambda_{3}-\lambda_{2}}{\eta}=0, \quad \mathrm{~V} \frac{\lambda_{1}-\lambda_{4}}{\theta}=0, . . .
$$

hold good, and show that the two other mutually opposite sides of the same inscribed quadrilateral, namely the sides $L_{2} L_{3}$, $\mathbf{L}_{4} \mathbf{L}_{1}$, are respectively parallel to the two vectors $\eta, \theta$, or to the axes of the two cylinders of revolution which can be circumscribed about the same original ellipsoid. Hence it is easy to infer the following theorem, which the author supposes to be new :-If on the mean axis $2 b$ of a given ellipsoid, abc, as the major axis, and with two foci $\mathrm{F}_{1}, \mathrm{~F}_{2}$, of which the common distance from the centre $\mathrm{A}$ is

$$
\overline{\mathbf{A F}}_{1}=\overline{\mathbf{A F}}_{2}=e=\frac{\sqrt[V]{ }\left(a^{2}-b^{2}\right) \sqrt{ }\left(b^{2}-c^{2}\right)}{\sqrt{ }\left(a^{2}-b^{2}+c^{2}\right)}, \ldots
$$

we construct an ellipsoid of revolution; and if, in any circular section of this new ellipsoid, roe inscribe a quadrilateral, $\mathrm{L}_{1} \mathbf{L}_{2} \mathbf{L}_{2} \mathbf{L}_{4}$, of which the two opposite sides $\mathbf{L}_{1} \mathbf{L}_{2}, \mathbf{L}_{3} \mathbf{L}_{4}$ are respectively parallel to the two umbilicar diameters of the given ellipsoid; rohile the two other and mutually opposite sides, $\mathrm{x}_{2} \mathrm{~L}_{3}, \mathrm{I}_{4} \mathrm{I}_{1}$, of the same inscribed quadrilateral, are respectively parallel to the axes of the two cylinders of revolution which can be circumscribed about the same given ellipsoid; then the point of intersection $\mathbf{E}$ of the first pair of opposite sides (namely of those parallel to the umbilicar diameters), roill be a point upon that given ellipsoid. It seems to the present writer that, in consequence of this remarkable relation between these two ellipsoids, the two foci $F_{1}, F_{2}$, of the above described ellipsoid of revolution, which have been seen to be situated upon the mean axis of the original ellipsoid, of which the three unequal semiaxes are denoted by $a, b, c$, may be not inconveniently called the Two MEDIAL FOCI of that original ellipsoid: but he gladly submits the question of the propriety of such a designation, to the judgement of other and better geometers. Meanwhile it may be noticed that the two ellipsoids intersect each other in a system of two ellipses, of which the planes are perpendicular to the axes of the two cylinders of revolution above mentioned; and that those four common tangent planes of the two ellipsoids, which are parallel to their common axis, that is to the mean axis of the original ellipsoid $a b c$, are parallel also to its two umbilicar diameters.

[To be continued.] 